\title{
Parteipolitisch zerstritten, national gespalten oder geschlossen gegen den Rat? Das Europäische Parlament und die $\mathrm{CO}_{2}$-Verordnung für Neuwagen
}

\author{
Marcelo Vollmann*
}

Die von der Europäischen Kommission im Dezember 2007 vorgeschlagene „Verordnung zur Festsetzung von Emissionsnormen für neue Personenkraftwagen" ${ }^{1}\left(\mathrm{CO}_{2}\right.$-Verordnung für Neuwagen) wurde im Rahmen des Energie- und Klimapakets durch das Europäische Parlament im Dezember 2008 in erster Lesung mit 559 Stimmen gegen 98 bei 60 Enthaltungen angenommen. ${ }^{2}$ Allerdings verdeckt dieses Ergebnis die während des Mitentscheidungsverfahrens sichtbar gewordenen Bruchlinien innerhalb des Parlaments. ${ }^{3}$ Ziel dieses Artikels ist eine Nachzeichnung der Willensbildung innerhalb des Europäischen Parlaments und ob diese parteipolitisch oder national gespalten erfolgte vor dem Hintergrund der Entstehungsgeschichte und eine Analyse der Faktoren, die zum letztendlichen Ergebnis geführt haben. Hierzu werden zuerst die Vorgeschichte, der Kommissionsvorschlag sowie die Interessenlage der Automobilhersteller und ,grünen“ Lobbyvereinigungen untersucht. Das folgende Kapitel skizziert und analysiert den Entscheidungsprozess mit Schwerpunkt auf dem Parlament. Parallel verlaufende wichtige Ereignisse, insbesondere in den Mitgliedstaaten und dem Ministerrat, werden in die Analyse integriert um ein Gesamtbild zu ermöglichen. Das letzte Kapitel schlägt eine Beurteilung für das Verhalten des Parlaments vor.

\section{Hintergrund und Vorgeschichte}

Die Auseinandersetzung um die $\mathrm{CO}_{2}$-Verordnung für Neuwagen muss vor dem Hintergrund der internationalen Klimaschutzvereinbarungen und der Vorgeschichte der $\mathrm{CO}_{2}$-Reduzierung bei Neuwagen begriffen werden. Alle Vertragsparteien der Klimarahmenkonferenz der Vereinten Nationen von 1993, deren Ergebnisse von der Europäischen Union angenommen worden sind, hatten sich verpflichtet, Maßnahmen zur Eindämmung des Klimawandels durch Verringerung ihrer Treibhausgasemissionen zu ergreifen. Im Jahre 2006 führte der „Sternreport“ und der ,Al Gore-Film“ „Eine unbequeme Wahrheit“ zu einer stärkeren Sensibilisierung der Öffentlichkeit in punkto Klimaschutz. Im Jahre 2007 entschied die Europäische Union, ihre Treibhausgasemissionen um mindestens 20 Prozent (30 Prozent im Falle eines internationalen Abkommens) verglichen mit dem Stand von 1990 bis zum Jahr 2020 zu reduzieren. Die $\mathrm{CO}_{2}$-Reduzierung sollte einerseits über die Revision des Emissionshandelssystems, andererseits über individuelle Reglementierungen von Industriesektoren erreicht werden. ${ }^{4}$

* Dr. Marcelo Vollmann, Umweltreferent bei Renault, Brüssel.

Dieser Artikel gibt nur die persönliche Meinung des Autors wieder.

1 Europäische Kommission: Vorschlag für eine Verordnung des Europäischen Parlaments und des Rates zur Festsetzung von Emissionsnormen für neue Personenkraftwagen im Rahmen des Gesamtkonzepts der Gemeinschaft zur Verringerung der $\mathrm{CO}_{2}$-Emissionen von Personenkraftwagen und leichten Nutzfahrzeugen, KOM (2007) 856.

2 Europäisches Parlament: Protokoll der Sitzung vom Mittwoch 17. Dezember 2008, Anlage Ergebnisse der Abstimmungen, P6_PV(2008)12-17, S. 15.

3 Europäisches Parlament: EP verabschiedet EU-Klimapaket, Pressemitteilung, 17.12.2008.

4 Europäische Kommission: Vorschlag für eine Verordnung zur Festsetzung von Emissionsnormen für neue Personenkraftwagen, 2007, S. 2. 
Die Europäische Kommission konkretisierte ihre Selbstverpflichtung durch das aus vier Vorhaben bestehende Energie- und Klimapaket: die Richtlinie über erneuerbare Energien, ${ }^{5}$ die Richtlinie über die Abtrennung und geologische Speicherung von $\mathrm{CO}_{2},{ }^{6}$ die Richtlinie über die dritte Phase des europäischen Emissionshandelssystems ${ }^{7}$ und die Entscheidung über die Lastenverteilung. ${ }^{8}$ Zwei weitere Rechtsetzungsvorhaben wurden parallel zu diesem Prozess initiiert und im Herbst 2008 integriert: zum einen die Richtlinie für Qualitätsstandards für Kraftstoffe ${ }^{9}$ und zum anderen die $\mathrm{CO}_{2}$-Verordnung für Neuwagen.

Der Vorschlag zur Festsetzung von $\mathrm{CO}_{2}$-Emissionsnormen für Neuwagen kann auf eine längere Entstehungsgeschichte zurückblicken. Die Kommission hatte erstmals 1995 eine Gemeinschaftsstrategie zur Minderung der $\mathrm{CO}_{2}$-Emissionen von Neuwagen angenommen. ${ }^{10}$ Diese gründete auf drei Pfeilern: eine Selbstverpflichtung der Automobilindustrie zur Senkung der Emissionen, bessere Informationen für die Verbraucher und steuerliche Maßnahmen für die Förderung sparsamer Autos. Der Kern der Strategie basierte auf der Selbstverpflichtung, da den steuerlichen Maßnahmen auf europäischer Ebene aus Souveränitätsgründen kein Erfolg beschieden sein sollte und die besseren Verbraucherinformationen zwar 1999 in einer Richtlinie konkretisiert wurden, aber kaum Wirkung zeigten.

Bei der Selbstverpflichtung visierte die Kommission das extrem ehrgeizige Ziel der Reduktion des $\mathrm{CO}_{2}$-Ausstoßes auf $120 \mathrm{~g} / \mathrm{km}$ für das Jahr 2005 an, allerdings sollte es sowohl in punkto Ziel als auch Zeitpunkt drei Änderungen geben. 1996 wurde das Datum für das Ziel durch die Umweltminister auf ,bis spätestens 2010‘ verschoben. 1998 erfolgte eine erneute Verschiebung auf das Jahr 2012 und der „Verband der Europäischen Automobilindustrie“ (ACEA) akzeptierte als Zwischenschritt, die durchschnittlichen $\mathrm{CO}_{2}$-Emissionen der verkauften Neuwagen um circa 25 Prozent auf 140 g/km bis 2008 zu reduzieren. Anfang 2007 überprüfte die Kommission die freiwillige Selbstverpflichtung und stellte fest, dass diese ohne zusätzliche Maßnahmen nicht erreicht werden konnte und kündigte an, einen Rechtsrahmen zur Verwirklichung des EU-Ziels von $120 \mathrm{~g} / \mathrm{km}$ vorzuschlagen. Allerdings wollte die Kommission den Automobilherstellern die Möglichkeit eröffnen, 10 g/km des Zieles mit anderen Mitteln als der Motorentechnik zu erreichen, nämlich mit sogenannten ergänzenden Maßnahmen wie beispielsweise effizienteren Klimaanlagen oder alternativen Kraftstoffen, was eine Abschwächung des Motorenzieles von $120 \mathrm{~g} / \mathrm{km}$ auf $130 \mathrm{~g} / \mathrm{km}$ erlaubte. ${ }^{11}$

5 Richtlinie 2009/28/EG des Europäischen Parlaments und des Rates vom 23. April 2009 zur Förderung der Nutzung von Energie aus erneuerbaren Quellen und zur Änderung und anschließenden Aufhebung der Richtlinien 2001/77/EG und 2003/30/EG, in: Amtsblatt der EU, Nr. L 140 vom 5. Juni 2009, S. 16-62.

6 Richtlinie 2009/31/EG des Europäischen Parlaments und des Rates vom 23. April 2009 über die geologische Speicherung von Kohlendioxid und zur Änderung der Richtlinie 85/337/EWG des Rates sowie der Richtlinien 2000/60/EG, 2001/80/EG, 2004/35/EG, 2006/12/EG und 2008/1/EG des Europäischen Parlaments und des Rates sowie der Verordnung (EG) Nr. 1013/2006, in: Amtsblatt der EU, Nr. L 140 vom 5. Juni 2009, S. 114-135.

7 Richtlinie 2009/29/EG des Europäischen Parlaments und des Rates vom 23. April 2009 zur Änderung der Richtlinie 2003/87/EG zwecks Verbesserung und Ausweitung des Gemeinschaftssystems für den Handel mit Treibhausgasemissionszertifikaten, in: Amtsblatt der EU, Nr. L 140 vom 5. Juni 2009, S. 63-87.

8 Entscheidung Nr. 406/2009/EG des Europäischen Parlaments und des Rates vom 23. April 2009 über die Anstrengungen der Mitgliedstaaten zur Reduktion ihrer Treibhausgasemissionen mit Blick auf die Erfüllung der Verpflichtungen der Gemeinschaft zur Reduktion der Treibhausgasemissionen bis 2020, in: Amtsblatt der EU, Nr. L 140 vom 5. Juni 2009, S. 136-148.

9 Richtlinie 2009/30/EG des Europäischen Parlaments und des Rates vom 23. April 2009 zur Änderung der Richtlinie 98/70/EG im Hinblick auf die Spezifikationen für Otto-, Diesel- und Gasölkraftstoffe und die Einführung eines Systems zur Überwachung und Verringerung der Treibhausgasemissionen sowie zur Änderung der Richtlinie 1999/32/EG des Rates im Hinblick auf die Spezifikationen für von Binnenschiffen gebrauchte Kraftstoffe und zur Aufhebung der Richtlinie 93/12/EWG, in: Amtsblatt der EU, Nr. L 140 vom 5. Juni 2009, S. 88-13.

10 Europäische Kommission: Vorschlag für eine Verordnung zur Festsetzung von Emissionsnormen für neue Personenkraftwagen, 2007, S. 2.

11 European Federation for Transport \& Environment: Reducing $\mathrm{CO}_{2}$ Emissions from New Cars: A Study of Major Car Manufacturers' Progress in 2007, August 2008, S. 6-7. 
ACEA und Umweltschutzorganisationen bewerteten diesen Prozess verständlicherweise unterschiedlich. Aus Sicht von ACEA war es nie gerechtfertigt von einem Versagen der Automobilindustrie bei der freiwilligen Selbstverpflichtung zu sprechen: Zum einen waren einige Sekundärziele wie die Vermarktung von Fahrzeugen unter $120 \mathrm{~g} / \mathrm{km}$ erreicht worden (16 Prozent Marktanteil im Jahre 2008) und zum anderen beeinträchtigten das Pkw-Gewicht erhöhende Faktoren, die außerhalb des Einflusses der Industrie lagen, wie zusätzliche europäische Gesetzgebung sowie ein Käufertrend zu sicheren Fahrzeugen, die Fortschritte der Industrie in der $\mathrm{CO}_{2}$-Reduzierung. ${ }^{12}$

Die beiden maßgeblich am Prozess beteiligten Umweltorganisationen „European Federation for Transport and Environment“ und „Greenpeace“ standen der Verschiebung des Zieldatums und des Motorenziels sehr kritisch gegenüber, da sie es nicht als ehrgeizig genug empfanden.

\section{Die Kernpunkte des Kommissionsvorschlages}

Das Ziel des Vorschlages war die Senkung der durchschnittlichen $\mathrm{CO}_{2}$-Emissionen der gesamten Neuwagenflotte im EU-Binnenmarkt im Jahre 2012 auf 130 g/km durch Motorentechnik im Rahmen eines Gesamtkonzeptes mit dem Oberziel, die durchschnittlichen $\mathrm{CO}_{2}$-Emissionen für Neuwagen im EU-Binnenmarkt im Jahr 2012 auf $120 \mathrm{~g} / \mathrm{km}$ zu reduzieren. Neben der Minderung auf durchschnittlich $130 \mathrm{~g} / \mathrm{km}$ durch Motorentechnik sollten weitere $10 \mathrm{~g} / \mathrm{km}$ durch sogenannte ,ergänzende Maßnahmen ‘ wie effizientere Klimaanlagen, Schaltpunktanzeigen, Reifendruckkontrollsysteme und Leichtlaufreifen sowie den Einsatz von Biokraftstoffen erreicht werden. Die $\mathrm{CO}_{2}$-Verordnung für Neuwagen war allerdings nur auf das $130 \mathrm{~g} / \mathrm{km}$ Ziel durch Motorentechnik im Jahr 2012 fokussiert, während die Details der ergänzenden Maßnahmen von $10 \mathrm{~g} / \mathrm{km}$ in anderen Rechtsetzungsverfahren geregelt werden sollen. Das $130 \mathrm{~g} / \mathrm{km}$ Ziel im Jahr 2012 bedeutet vor dem Hintergrund einer durchschnittlichen $\mathrm{CO}_{2}$-Emission der Neuwagenflotte in der Europäischen Union von circa 160 g/km im Jahre 2006 eine durchschnittlich notwendige Reduzierung der $\mathrm{CO}_{2}$-Emissionen von 19 Prozent für alle Neuwagen.

Da die Kommission sich entschieden hatte, den $\mathrm{CO}_{2}$-Ausstoß an die Masse (,Fahrzeuggewicht ${ }^{* 13}$ ) der Neuwagen zu binden, lautete die entscheidende Frage: Welcher Hersteller muss seinen Flottendurchschnitt für Neuwagen um wie viel reduzieren? In der Konsequenz hieß dies unterschiedliche Emissionsziele für jeden Hersteller, je nach Gewicht seiner Neuwagenflotte; mit der Folge, je höher das Durchschnittsgewicht der Neuwagen eines Herstellers, desto höher war der erlaubte Emissionswert. Dies galt natürlich auch umgekehrt. Ein Hersteller leichterer Fahrzeuge bekäme einen niedrigeren Emissionswert vorgeschrieben. Diese Lastenverteilung auf Basis des Fahrzeuggewichts war unter den Herstellern vor dem Hintergrund ihrer unterschiedlich schweren Fahrzeugsegmente umstritten.

Im Falle, dass ein Automobilhersteller seine Zielvorgabe nicht einhalten sollte, droht ihm eine aus drei Faktoren zu berechnende Strafzahlung: die Überschreitung der Zielvorgabe in $\mathrm{g} / \mathrm{km}$ multipliziert mit der Strafhöhe pro überschrittenem $\mathrm{g} / \mathrm{km}$ multipliziert mit der Anzahl der in dem Jahr neu zugelassenen Neuwagen des Herstellers. Die Kommission ließ die Strafhöhe dabei über die Jahre 2012 bis 2015 ansteigen: 20 Euro pro verfehltem Gramm in 2012, 35 Euro in 2013, 60 Euro in 2014 und 95 Euro ab dem Jahr 2015. ${ }^{14}$

12 Verband der Europäischen Automobilindustrie: ACEA 1998 Commitment has Significantly Cut CO2 Emissions from Cars, Pressemitteilung.

13 In diesem Artikel wird die ,Masse“ des Fahrzeugs dem besseren Verständnis wegen als ,Fahrzeuggewicht ' bezeichnet.

14 Europäische Kommission: Vorschlag für eine Verordnung zur Festsetzung von Emissionsnormen für neue Personenkraftwagen, 2007. 


\section{Die Interessenlage der Automobilhersteller und Umweltschutzorganisationen}

Die Automobilindustrie akzeptierte die technische Herausforderung der $\mathrm{CO}_{2}$-Reduzierung, beanstandete aber die unausgewogene Balance des Kommissionsvorschlages, der dem Umweltschutz vor der Wettbewerbsfähigkeit der Industrie den Vorzug gab. Grundsätzlich hielt die Industrie den von ihr favorisierten integrierten Ansatz nicht genügend berücksichtigt. Dieser sah vor, neben der Motortechnik auch einen Beitrag der Kraftstoffindustrie durch Biokraftstoffe, der Fahrer durch ökologisches Fahren und der Mitgliedstaaten durch eine Besteuerung von $\mathrm{CO}_{2}$ sowie Infrastrukturmaßnahmen zur $\mathrm{CO}_{2}$-Reduzierung zu fordern. Darüber hinaus kritisierte die Automobilindustrie drei Elemente des Kommissionsvorschlages: ${ }^{15}$

Erstens die zu früh geforderte Implementierung der Verordnung. Denn nur neu zu entwickelnde Fahrzeuge könnten technisch in Bezug auf den $\mathrm{CO}_{2}$-Ausstoß noch geändert werden. Die Konzeption eines Modells dauert aber circa fünf bis sieben Jahre und dieses Modell wird dann über mehrere Jahre produziert. Die meisten der 2012 zu verkaufenden Neuwagen befanden sich bereits weit fortgeschritten in der Entwicklung. Daher beharrte die Industrie auf Planungssicherheit und eine mit diesem industriellen Produktzyklus zu vereinbarende Verordnung, was ein Inkrafttreten im Jahr 2012 durch eine erst im Jahr 2009 abgeschlossene Rechtsetzung unmöglich mache. Der ACEA mahnte eine gestaffelte Einführung (,phase-in`) der Verordnung über mehrere Jahre an. Nur ein Teil der Neuwagenflotte sollte anfänglich der $\mathrm{CO}_{2}$-Verordnung für Neuwagen unterliegen und dieser Anteil würde dann schrittweise auf 100 Prozent erhöht.

Außerdem forderte der ACEA, dass weitere Anreize für die Entwicklung von $\mathrm{CO}_{2}$ reduzierenden Technologien, sogenannten Ökoinnovationen, in die Verordnung aufgenommen werden sollten. Diese messbar $\mathrm{CO}_{2}$ verringernden Technologien, deren Reduktion aber noch nicht im derzeitig verwendeten europäischen Testverfahren zur $\mathrm{CO}_{2}$-Ermittlung abgebildet wird, sollten ebenfalls als $\mathrm{CO}_{2}$-Senkung auf die Zielvorgabe der Hersteller anrechenbar sein. Ein Beispiel für eine Ökoinnovation wären energieeffizientere Scheinwerfer.

Drittens kritisierte die Industrie die immense Höhe der Strafzahlungen, da diese bereits bei einer geringfügigen Verfehlung ruinöse Kosten von mehreren 100 Millionen Euro zur Folge haben könnten, wogegen für andere in den europäischen Emissionshandel integrierte Industriesektoren deutlich niedrigere Strafzahlungen für das Überschreiten der Ziele bei der Reduktion von $\mathrm{CO}_{2}$-Emissionen angesetzt waren. ${ }^{16}$

Die Industrie präsentierte sich in dem politischen Prozess weitgehend geschlossen, obwohl es Differenzen zwischen den Herstellern gab: Am umstrittensten war die vorgeschlagene Lastenverteilung der Kommission mit der Bindung der $\mathrm{CO}_{2}$-Ziele für jeden Hersteller an das Fahrzeuggewicht. Zum besseren Verständnis muss man sich die Marktstruktur vergegenwärtigen: Die französischen und italienischen Automobilhersteller sind stark im preisgünstigeren, leichteren und weniger $\mathrm{CO}_{2}$ ausstoßenden Kleinwagensegment vertreten, während insbesondere die deutschen Hersteller von Premiummarken, wie „Daimler“, „BMW“ und „Audi“, teurere, schwerere und mehr $\mathrm{CO}_{2}$ ausstoßende Fahrzeuge produzieren. Dabei ist zu berücksichtigen, dass der Gewinn im Premiumsegment deutlich höher als im Volumengeschäft ist.

Die Bindung der individuellen Zielvorgaben der Hersteller an das Fahrzeuggewicht führt bei einem politisch vorgegebenen zu erreichenden Durchschnittsziel von $130 \mathrm{~g} / \mathrm{km}$ dazu,

15 Rede des ACEA-Präsidenten Christian Streiff in Brüssel am 26.02.2008.

16 Ebenda. 
dass die Hersteller unterschiedlich strenge Ziele für das Jahr 2012 erhalten werden (unter der Annahme, dass das Fahrzeuggewicht ihrer Neuwagen sich zwischen 2007 und 2012 nicht ändert): $122 \mathrm{~g} / \mathrm{km}$ für „Fiat“, $127 \mathrm{~g} / \mathrm{km}$ für die französischen Hersteller und „Toyota“, $129 \mathrm{~g} /$ km für „General Motors“, 132 g/km für „Ford“, 133 g/km für „Volkswagen“, 137 g/km für „BMW“" und „Daimler“. 17

Allerdings unterschied sich auch der durchschnittliche $\mathrm{CO}_{2}$-Ausstoß der europäischen Neuwagenflotten im Jahre 2006 deutlich voneinander: 142 g/km bei „Peugeot“/,Citroën“, $144 \mathrm{~g} / \mathrm{km}$ bei „Fiat“, $147 \mathrm{~g} / \mathrm{km}$ bei „Renault“, 153 g/km bei „Toyota“, 157 g/km bei „,General Motors“, 162 g/km bei „Ford“, 166 g/km bei „Volkswagen“, 184 g/km bei „BMW“, 188 $\mathrm{g} / \mathrm{km}$ bei „Daimler“. Im Jahre 2006 lag der durchschnittliche $\mathrm{CO}_{2}$-Ausstoß der deutschen Hersteller „Volkswagen“, „Daimler“ und „BMW“ bei 173 g/km, der japanischen Automobilhersteller bei $161 \mathrm{~g} / \mathrm{km}$ und der französischen Automobilhersteller bei $144 \mathrm{~g} / \mathrm{km} .^{18}$

Die Umweltschutzorganisationen begrüßten die verbindlichen Vorgaben, die die in ihren Augen unwirksame freiwillige Selbstverpflichtung ersetzte. Allerdings forderten sie ein Motorenziel von $120 \mathrm{~g} / \mathrm{km}$ und nicht von $130 \mathrm{~g} / \mathrm{km}$ für das Jahr 2012. Auch hätten sie als Parameter die Fahrzeugstandfläche, die der Fläche zwischen den vier Rädern entspricht, anstelle des Fahrzeuggewichts bevorzugt, um den Herstellern einen Anreiz zum Reduzieren des Fahrzeuggewichts zu setzen. Darüber hinaus verlangten die Umweltschutzorganisationen als langfristiges Ziel einen $\mathrm{CO}_{2}$-Ausstoß von $80 \mathrm{~g} / \mathrm{km}$ für das Jahr 2020, höhere Strafzahlungen von 150 Euro je überschrittenem $\mathrm{g} / \mathrm{km}$, keinerlei Ausnahmen und befürworteten eine Höchstgrenze von $\mathrm{CO}_{2}$-Emissionen für einzelne Autos. ${ }^{19}$

\section{Der Entscheidungsprozess}

\section{Spezifika des Entscheidungsprozesses}

Das Europäische Parlament besaß im Zuge des Mitentscheidungsverfahrens das gleiche Mitspracherecht wie der Ministerrat und verfügte über ein Vetorecht. Allerdings unterlag der Entscheidungsprozess im Parlament den folgenden spezifischen Elementen:

Erstens einem starken Zeitdruck: Die Kommission hatte im Dezember 2007 ihren Vorschlag präsentiert, es standen allerdings bereits im Juni 2009 Wahlen zum Europäischen Parlament und im Herbst 2009 die Bildung einer neuen Kommission an. Da ein gewöhnliches Mitentscheidungsverfahren circa zwei Jahre dauert, bedeutete dies die Notwendigkeit eines schnellen Verfahrens, das allerspätestens im Frühjahr 2009 abgeschlossen sein musste.

Zweitens der Notwendigkeit einer Einigung in erster Lesung mit dem Rat: Aufgrund des Zeitdrucks mussten die europäischen Institutionen das Verfahren in erster Lesung abschlieBen. Dies bedeutete, dass das Parlament auf Basis der Beratungen des Umweltausschusses mit dem Rat informell verhandeln musste, um in der Abstimmung im Plenum das Endergebnis beschließen zu können. Der Ministerrat musste seinerseits, parallel zum Willensbildungsprozess innerhalb des Parlaments, versuchen, eine gemeinsame Position zu finden.

Drittens einer Ausschusskonfiguration mit verstärkter Zusammenarbeit: Das Plenum verwies die $\mathrm{CO}_{2}$-Verordnung für Neuwagen federführend an den Ausschuss für Umweltfragen,

17 European Federation for Transport and Environment: Reducing $\mathrm{CO}_{2}$ Emissions from new cars, 2008, S. 11. Entscheidend ist hier nicht ob die einzelnen Werte auf das Gramm exakt stimmen, sondern dass gezeigt wird, dass die verschiedenen Hersteller unterschiedliche Zielvorgaben aufgrund des verschiedenen Gewichts ihrer Fahrzeuge erhalten.

18 Ebenda, S. 9.

19 Greenpeace: Driving Climate Change. How the car industry is lobbying to undermine EU fuel efficiency legislation, Amsterdam 2008, S. 7. 
Volksgesundheit und Lebensmittelsicherheit, der Ausschuss für Industrie, Forschung und Energie und der Ausschuss für Verkehr und Fremdenverkehr kündigten Stellungnahmen an. Die Bedeutung des Industrieausschusses wurde aber durch die Anwendung der verstärkten Zusammenarbeit mit dem Umweltausschuss entscheidend aufgewertet, die ihm Einfluss auf den Zeitplan, bessere Unterrichtung durch den federführenden Ausschuss und ausschließliche beziehungsweise gemeinsame Zuständigkeit über in seinen Bereich fallende Teile des Textes zugestand. Letzteres soll nicht im Einzelnen geklärt werden, bedeutete aber eine machtpolitische Aufwertung des Industrieausschusses und sollte zu zwei unterschiedlichen Meinungsbildungsprozessen in den beiden Ausschüssen des Parlaments führen.

\section{Meinungsbildung im Parlament}

Unterschiedliche Auffassungen im Parlament waren bereits in zwei Berichten kurz vor und nach der Veröffentlichung des Kommissionsvorschlages deutlich geworden. Im Oktober 2007 begrüßte das Europäische Parlament in einer nicht bindenden umweltfreundlichen Entschließung („Davies Report“) ${ }^{20}$ des damaligen britischen, liberalen Berichterstatters Chris Davies die Absicht der Kommission, eine Verordnung zu erlassen, plädierte aber für ein Motorenziel von $125 \mathrm{~g} / \mathrm{km}$ für das Jahr 2015, den Parameter Fahrzeugstandfläche und verlangte darüber hinaus als langfristiges Ziel die Marke von $95 \mathrm{~g} / \mathrm{km}$ für das Jahr 2020.

Im Januar 2008 bestätigte das Europäische Parlament zwar diese Zielsetzung in einer anderen, das gesamte Kfz-Regelungssystem unter Wettbewerbsaspekten analysierenden Entschließung (,CARS 21 Bericht“). ${ }^{21}$ Allerdings intonierte dieser Text des deutschen, liberalen Berichterstatters Jorgo Chatzimarkakis eine industriefreundlichere Melodie indem er ein langfristiges Ziel forderte, aber nicht spezifizierte, der Automobilindustrie genügend Zeit für die notwendigen Anpassungen lassen wollte und allgemein die Bedeutung der Wettbewerbsfähigkeit der Automobilindustrie unterstrich. Dazu befürwortete der Berichterstatter den von der Kommission vorgeschlagenen Parameter Fahrzeuggewicht.

Beide Auffassungen sollten auch in der personellen Konstellation im Parlament bei den Beratungen der $\mathrm{CO}_{2}$-Verordnung für Neuwagen wieder auftauchen: Berichterstatter im Umweltausschuss wurde der italienische Sozialist Guido Sacconi. Die Schattenberichterstatter waren der britische Konservative Martin Callanan, der britische Liberale Chris Davies sowie die deutsche Grüne Rebecca Harms. Im Industrieausschuss übernahm der deutsche Christdemokrat Werner Langen die Berichterstatterrolle, der ebenfalls Vorsitzender der CDU/ CSU-Gruppe im Europäischen Parlament ist. Schattenberichterstatter wurden der französische Sozialist Pierre Pribetich, der deutsche Liberale Jorgo Chatzimarkakis und wiederum die deutsche Grüne Rebecca Harms, die somit in beiden Ausschüssen involviert war. Im Transportausschuss wurde der Ire Seán Ó Neachtain Berichterstatter, der der kleinen „Fraktion Union für das Europa der Nationen“ (UEN) angehörte.

Die Debatten ab April 2008 in den Ausschüssen offenbarten die Positionen der Abgeordneten und Fraktionen: Die deutschen Liberalen und Konservativen setzten sich stark für die Einführung von Ökoinnovationen ein. Die meisten Liberalen und Konservativen unterstützten niedrigere Strafen und eine zeitlich gestaffelte Einführung der Verordnung. Die Grünen befürworteten dagegen höhere Strafen, härtere Ziele und keine Ausnahmen. Der Parameter Fahrzeuggewicht und die Lastenverteilung wurden von den Grünen, den Sozialdemokraten

20 Europäisches Parlament: Strategie der Gemeinschaft zur Minderung der $\mathrm{CO}_{2}$-Emissionen von Personenkraftwagen und leichten Nutzfahrzeugen, Entschließung P6_TA(2007)0469 vom 24.10.2007.

21 Europäisches Parlament: CARS 21: Ein wettbewerbsfähiges Kfz-Regelungssystem für das 21. Jahrhundert, Entschließung P6_TA(2008)0007 vom 15.01.2008. 
und französischen Konservativen angegriffen. Einige deutsche Liberale und Konservative sprachen sich ebenso für eine Änderung der Lastenverteilung zugunsten der schweren Fahrzeuge aus.

\section{Die Verhandlungen im Ministerrat bis zur deutsch-französischen Einigung im Juni 2008}

Der Umweltrat am 3. März 2008 in Brüssel führte zu einer ersten großen Debatte unter den Mitgliedstaaten auf Ebene der Umweltminister, in der sich 18 der 27 Länder äußerten. Dabei zeigten sich drei unterschiedliche Lager:

Erstens Länder, die Produzenten schwerer Fahrzeuge oder deren Zulieferer beheimaten, wie Deutschland, Schweden, Österreich, Ungarn und Tschechien, verlangten eine bessere Lastenverteilung zugunsten der schwereren Fahrzeuge. Der Kommissionsvorschlag hatte insbesondere in deutschen Regierungskreisen eine harte Reaktion provoziert: „Vernichtungsfeldzug gegen deutsche Autoindustrie“ (Wirtschaftsminister Michael Glos), ,,industriepolitisch motivierter Angriff auf die deutsche Autoindustrie“ (Umweltminister Sigmar Gabriel). ${ }^{22}$

Zweitens Länder, die Standort für Produzenten leichterer Fahrzeuge oder deren Zulieferer sind, wie Frankreich, Italien und Rumänien, forderten eine Lastenverteilung zugunsten der dort ansässigen Automobilindustrie und zeigten teils auch Sympathie für eine Streichung des Parameters Fahrzeuggewicht zugunsten eines Parameters Fahrzeugstandfläche.

Drittens forderten Länder ohne Automobilindustrie eine stärker umweltfreundliche Ausrichtung. Dazu gehörten Staaten wie die Niederlande, Dänemark oder Irland, die neben höheren Strafen, einen anderen Parameter statt des Fahrzeuggewichts und insbesondere ein langfristiges Ziel für das Jahr 2020 forderten.

Großbritannien nahm eine spezielle Position ein: Es unterstützte als langfristiges Ziel die Reduktion des $\mathrm{CO}_{2}$-Ausstoßes auf $100 \mathrm{~g} / \mathrm{km}$ ab 2020, forderte darüber hinaus aber Ausnahmeregelungen für sogenannte Nischenhersteller mit kleineren Produktionsvolumina, die sich natürlich in erster Linie in Großbritannien befinden („Land Rover“ und „Jaguar").

Polen, Tschechien und die Slowakei sprachen sich dagegen für ein späteres Inkrafttreten der Verordnung aus, um der Industrie mehr Zeit für die Anpassung zu geben: 2015 statt 2012.

Insgesamt zeichneten sich also zwei Frontlinien im Ministerrat ab: einerseits zwischen den Ländern, die Hersteller schwerer Fahrzeuge beheimaten, und jenen, in denen Kleinwagenhersteller ansässig sind, insbesondere in der Frage der Lastenverteilung. Beide Lager waren aber stark genug, um jegliche Änderung des Kommissionsvorschlages zu verhindern. Andererseits standen umweltpolitische gegen industriepolitische Interessen: Umweltpolitisch erhielt die Einführung eines langfristigen Ziels für 2020 Unterstützung. Die Industrie konnte dagegen Rückendeckung für ihr Anliegen einer Reduzierung der Strafen und einer stufenweisen Einbeziehung der Neuwagenflotte in die Regulierung verbuchen.

Bilaterale deutsch-französische Gespräche hatten in der ersten Hälfte 2008 keine Annäherung gebracht. ${ }^{23}$ Die Kommission hatte im April 2008 deutlich gemacht, dass ihr die Lastenverteilung gleichgültig sei, solange das Gesamtziel erreicht werde. ${ }^{24}$ Allerdings sollte ein Treffen von Angela Merkel und Nicolas Sarkozy am 9. Juni 2008 in Straubing den deutschfranzösischen ,Autostreit‘ entschärfen: Beide Seiten unterstützten den Vorschlag der Kommission bezüglich der Zielsetzung und der Lastenverteilung zwischen den Herstellern mit

22 Tagesschau.de: Drastische Reaktionen auf EU-Pläne zur $\mathrm{CO}_{2}$ Minderung, 21.12.2007.

23 Helmut Hauschild/Klaus Stratmann: Abgasstreit um Autos eskaliert, in: Handelsblatt 11.-13.04.2008.

24 Handelsblatt: Dimas geht im $\mathrm{CO}_{2}$-Streit auf deutsche Autobauer zu, 16.04.2008. 
der Entscheidung für den Parameter Fahrzeuggewicht. Das langfristige Ziel für das Jahr 2020 sollte zwischen 95 und 110 g/km liegen, aber auf Grundlage einer gründlichen Folgenabschätzung definiert werden. Weiterhin sollte es eine schrittweise Einbeziehung der Neuwagenflotte (,phase-in') in die Regulierung geben. Die Strafen bei Verfehlung der Ziele sollten bei einer geringeren Verfehlung niedriger ausfallen und die Anrechnung von Ökoinnovationen auf 6-8 $\mathrm{g}$ beschränkt werden. ${ }^{25}$

Der deutsch-französische Kompromiss sollte die Streitigkeiten um den Parameter und die Lastenverteilung weitgehend beenden und dem Parlament in den anderen Punkten mögliche Kompromisslinien aufzeigen.

\section{Konfliktlinien im Parlament}

Die verschiedenen Interessen der unterschiedlichen Akteure zwischen Konservativen, Liberalen und Sozialisten lassen sich an den Diskussionen der Ausschüsse und den eingebrachten Änderungsanträgen zu den Rechtsetzungsvorhaben ablesen:

Die Festlegung eines langfristigen Ziels für das Jahr 2020 kristallisierte sich als Priorität der politisch linken Hälfte des Parlaments heraus, was grundsätzlich auch von den Liberalen und Konservativen unterstützt wurde. Allerdings gab es unterschiedliche Auffassungen über die Frage einer Revisionsklausel im Jahr 2014: Sollte diese auch das Ziel ändern können oder nur die Modalitäten zum Erreichen des Ziels behandeln?

Die Konservativen und Teile der Liberalen verlangten eine schrittweise Einbeziehung der Neuwagenflotte (,phase-in') in die Verordnung und eine Reduzierung der hohen Strafen. Der Berichterstatter Guido Sacconi unterstrich dagegen, dass er nur einen ,phase-in ${ }^{\text {' } z w i-}$ schen 2012 und 2015 akzeptieren würde: entweder eine schrittweise Einbeziehung der Neuwagenflotte oder eine wie auch immer modifizierte Strafregelung.

Eine Einigung für eine schrittweise Einbeziehung der Neuwagenflotte in den vier Jahren von 2012 bis 2015 verlangte auch konkrete Prozentsätze: Die Konservativen, Liberalen und Sozialdemokraten unterstützten unterschiedliche Modelle (siehe Tabelle).

Tabelle: Positionen von wichtigen Abgeordneten der jeweiligen Fraktionen zur schrittweisen Einbeziehung der Neuwagenflotte von 2012 bis 2015

\begin{tabular}{|l|c|c|c|c|}
\hline Fraktion & $\mathbf{2 0 1 2}$ & $\mathbf{2 0 1 3}$ & $\mathbf{2 0 1 4}$ & $\mathbf{2 0 1 5}$ \\
\hline Konservative & 25 Prozent & 50 Prozent & 75 Prozent & 100 Prozent \\
\hline Liberale & 40 Prozent & 60 Prozent & 80 Prozent & 100 Prozent \\
\hline Sozialdemokraten & 70 Prozent & 80 Prozent & 90 Prozent & 100 Prozent \\
\hline
\end{tabular}

Quelle: Eigene Darstellung.

Konservative und Liberale wollten die Strafen in unterschiedlicher Höhe reduzieren, während die Sozialdemokraten höhere Strafen unterstützten.

Die Lastenverteilung und die Parameterfrage standen dagegen seit der Einigung zwischen Nicolas Sarkozy und Angela Merkel nicht mehr im Mittelpunkt, wenngleich es weiter

25 Deutsch-Französischer Ministerrat: Deutsch-französische Erklärung zum Schadstoffausstoß von Personenkraftwagen, Straubing, 09.06.2008. 
Anträge von französischen Sozialdemokraten, italienischen und deutschen Konservativen gab, sie zugunsten der jeweiligen eigenen Hersteller zu ändern.

Bei den Strafzahlungen verlangten die französischen, rumänischen, slowakischen und italienischen Konservativen, die französischen, spanischen und rumänischen Sozialdemokraten sowie die rumänischen Liberalen neben der oben angesprochenen Reduzierung der absoluten Beträge, die Nichtberücksichtigung von weniger $\mathrm{CO}_{2}$ ausstoßenden Fahrzeugen bei der Berechnung der Strafzahlungen. Diese Maßnahme hätte allen Herstellern geholfen, aber natürlich stärker den Produzenten der wenig $\mathrm{CO}_{2}$ ausstoßenden Neuwagen.

Limitierte Ökoinnovationen konnten auf eine breite Mehrheit im Parlament zählen, wenngleich die Unterstützung dafür bei den deutschen Abgeordneten aus der „Allianz der Liberalen und Demokraten für Europa“ (ALDE) und der damaligen ,Fraktion der Europäischen Volkspartei (Christdemokraten) und europäischer Demokraten“ (EVP-ED) größer war.

Die Ausnahmeregelung für Nischenhersteller von 10.000 bis 300.000 Fahrzeugen wurde von liberalen, konservativen und sozialdemokratischen britischen Abgeordneten gemeinsam eingebracht.

Die Grünen, insbesondere die deutschen mit ihrer, Wortführerin in Autofragen ' Rebecca Harms, und die weiter links positionierte „Konföderale Fraktion der Vereinigten Europäischen Linken/Nordische Grüne Linke“" warben aus Umweltschutzgründen für eine härtere Linie gegenüber der Automobilindustrie: Sie forderten ein schärferes Ziel (120 g/km Motorenziel statt $130 \mathrm{~g} / \mathrm{km}$ ), einen anderen Parameter (Fahrzeugstandfläche statt Fahrzeuggewicht), eine bessere Lastenverteilung für Produzenten leichterer und $\mathrm{CO}_{2}$ ärmerer Autos, höhere Strafen (150 Euro statt 95 Euro), keine Ausnahmen für Nischenhersteller, keine Anrechnung von Ökoinnovationen, da sie das Motorenziel unterliefen, und ein ehrgeiziges Ziel für 2020 von $80 \mathrm{~g} / \mathrm{km}$. Hauptangriffspunkt waren die viel $\mathrm{CO}_{2}$ ausstoßenden Modelle der deutschen Automobilindustrie. ${ }^{26}$

\section{Die Abstimmungen in den Ausschüssen}

Am 25. Juni 2008 scheiterte die Annahme einer Stellungnahme des der kleinen UEN angehörenden Berichterstatters Ó Neachtain ${ }^{27}$ im Verkehrsausschuss. Zuerst wurden die Änderungsvorschläge einzeln abgestimmt: Die Konservativen verloren die Abstimmung um den ,phase-in' mit 25 zu 15 Stimmen, die Sozialdemokraten scheiterten mit der Durchsetzung eines Motorenziels von $120 \mathrm{~g} / \mathrm{km}$ (anstatt $130 \mathrm{~g} / \mathrm{km}$ wie im Kommissionsvorschlag) in 2012 und einem langfristigen Ziel von 80 g/km ab 2020 mit 23 gegen 16 Stimmen. Daher ließen beide Gruppierungen die Stellungnahme mit 28 gegen 10 Stimmen durchfallen. Dieses Votum sollte nicht überbewertet werden, zeigte aber die sehr umweltfreundliche Ausrichtung der Sozialdemokraten in diesem Ausschuss wie auch die Unmöglichkeit der Fraktionen, sich zu diesem Zeitpunkt auf einen tragfähigen Kompromiss zu einigen.

26 Europäisches Parlament: Entwurf einer Stellungnahme des Ausschusses für Industrie, Forschung und Energie für den Ausschuss für Umweltfragen, Volksgesundheit und Lebensmittelsicherheit, PE404.748v01-00 vom 08.05.2008, Berichterstatter: Werner Langen; Europäisches Parlament: Änderungsanträge 47-194. Entwurf einer Stellungnahme Werner Langen, PE 407.863v01-00 vom 17.06.2008; Europäisches Parlament: Entwurf eines Berichts des Ausschusses für Umweltfragen, Volksgesundheit und Lebensmittelsicherheit, PE 406.014v01-00 vom 08.05.2010, Berichterstatter: Guido Sacconi; Europäisches Parlament: Änderungsanträge 11-265. Entwurf eines Berichts Guido Sacconi, PE 407.904v01-00 vom 18.06.2008.

27 Europäisches Parlament: Entwurf einer Stellungnahme des Ausschusses für Verkehr und Fremdenverkehr für den Ausschuss für Umweltfragen, Volksgesundheit und Lebensmittelsicherheit, PE 404.814v01-00 vom 30.04.2008, Berichterstatter: Seán Ó Neachtain; Europäisches Parlament: Änderungsanträge 20-82. Entwurf einer Stellungnahme Seán Ó Neachtain, PE 407.715v01-00 vom 05.06.2008. 
Am 1. September 2008 stimmte eine liberal-konservative Mehrheit im Industrieausschuss für die Kompromissvorschläge des Berichterstatters Werner Langen, der einige Industrieund Umweltanliegen miteinander verband: eine stufenweise Einbeziehung der Neuwagenflotte mit Schritten von 60, 70, 80 und 100 Prozent zwischen 2012 und 2015 (Abstimmungsergebnis: 34 zu 22); eine Reduzierung der Strafen auf 40 Euro je überschrittenem g/ $/ \mathrm{km} \mathrm{CO}_{2}$ Ausstoß; die Nichtberücksichtigung von wenig $\mathrm{CO}_{2}$ ausstoßenden Fahrzeugen bei der Berechnung der Strafzahlungen, die Einführung der Anrechnung von Ökoinnovationen, wenn auch nur in einem limitierten Umfang von sieben Gramm und die Einführung von Ausnahmen für die in Großbritannien beheimateten Nischenhersteller. Allerdings beinhaltete der Kompromiss auch das von der Industrie als extrem ehrgeizig gesehene langfristige Ziel von $95 \mathrm{~g} / \mathrm{km}$ ab 2020. Der Bericht wurde allerdings nur mit 35 zu 21 Stimmen bei 2 Enthaltungen angenommen, was als Zeichen eines starken Protestes bewertet werden kann.

Im Vorfeld der wichtigsten Abstimmung im Umweltausschuss am 25. September 2008 hieß es, dass es zu einem Kompromiss zwischen Konservativen und Sozialdemokraten gekommen sei. Das „Handelsblatt“ meldete noch am Tag der Abstimmung Details: ,phase-in“ mit Stufen von 70, 70, 80 und 100 Prozent, 50 Euro Strafe je überschrittenem g/km, Ökoinnovationen. ${ }^{28}$ Allerdings entwickelten die Fraktionen ein Eigenleben. Der , phase-in' und die niedrigeren Strafen wurden mit 23 Ja-, 39 Neinstimmen und 2 Enthaltungen abgelehnt. Neben den Grünen und der ,Konföderalen Fraktion der Vereinigten Europäischen Linken/Nordische Grüne Linke" hatten auch die meisten Liberalen und einige Konservative dagegen gestimmt. ${ }^{29}$ Am Ende stand jedenfalls ein sehr umweltfreundlicher Bericht, der in Grundzügen wieder den Kommissionsvorschlag einsetzte, wenngleich die Ausnahmen für Nischenhersteller sowie Ökoinnovationen gebilligt wurden. In der Endabstimmung konnten die 19 Gegenstimmen nichts gegen die 46 Befürworter ausrichten, zeigten aber wiederum die unterschiedlichen Auffassungen des Parlaments auf. Der Umweltausschuss setzte dem Ergebnis des Industrieausschusses ein Resultat mit einer stärkeren Umweltorientierung entgegen.

Das Parlament hatte in drei Abstimmungen seine Zerrissenheit offenbart und ging mit zwei widersprüchlichen Ergebnissen aus dem Industrie- und Umweltausschuss in die Verhandlungen mit dem Rat. Natürlich wollte der Industrieausschuss nicht akzeptieren, dass ausschließlich der federführende Umweltausschuss mit dem Rat verhandelte und letztendlich wurden die Ausschussvorsitzende und der Berichterstatter des Industrieausschusses in das Verhandlungsteam des Parlaments aufgenommen.

\section{Die Verhandlungen zwischen Parlament und Rat}

Die Verhandlungen mit dem Rat standen unter massivem Zeitdruck, weil die französische Präsidentschaft alle umweltrelevanten Rechtsetzungsvorhaben verabschieden wollte. Die $\mathrm{CO}_{2}$-Verordnung für Neuwagen war gemeinsam mit der Richtlinie für Qualitätsstandards für Kraftstoffe gegen Ende 2008 ebenfalls dem Klimapaket zugeschlagen worden, um den Verhandlungsdruck zwischen Rat und Parlament für eine Einigung bis Ende 2008 und mit Blick auf die internationalen Klimakonferenzen im Jahr 2009 zu erhöhen, den Erfolg der ursprünglich - vor Georgienkrieg und Finanzkrise - im Zeichen der Umwelt stehenden französischen Präsidentschaft zu verdeutlichen und die internationale Führungsrolle der Europäischen Union in der Klimaschutzfrage zu unterstreichen.

Letztendlich einigten sich die Institutionen auf einen Kompromiss, der am 17. Dezember 2008 vom Parlament in erster Lesung mit überwältigender Mehrheit angenommen wurde:

28 Helmut Hauschild: Lösung im $\mathrm{CO}_{2}$-Autostreit in Sicht, in: Handelsblatt, 25.09.2008.

29 Roll-call-votes des Europäischen Parlaments bei der Abstimmung am 25.09.2008. 
einen ,phase-in " mit Stufen von 65, 75, 80 und 100 Prozent, Strafen von 95 Euro je g/km $\mathrm{CO}_{2}$-Ausstoß über der individuellen Zielvorgabe (des Automobilherstellers) ab dem Jahr 2019 , davor aber für das erste bis dritte $\mathrm{g} / \mathrm{km} \mathrm{CO}_{2}$-Ausstoß über der individuellen Zielvorgabe nur 5, 15 beziehungsweise 25 Euro Strafe und 95 Euro ab dem vierten $\mathrm{g} / \mathrm{km}$ dazu die Anrechnung von $7 \mathrm{~g} / \mathrm{km}$ Ökoinnovationen auf die individuelle Zielvorgabe und Ausnahmeregelungen für Nischenhersteller. Dazu ein langfristiges Ziel von $95 \mathrm{~g} / \mathrm{km}$ ab 2020, das im Jahr 2014 lediglich im Hinblick auf die Modalitäten seiner Erreichung überprüft werden soll. Aus Industriesicht sind insbesondere das extrem ehrgeizige langfristige Ziel für 2020 und die nach wie vor hohen Strafzahlungen, die es in anderen Sektoren nicht in dieser Höhe gibt, schwer zu kritisieren. Die , grünen` Umweltverbände hätten dagegen eine schärfere Gesetzgebung befürwortet.

\section{Analyse des Verhaltens des Parlaments}

Das Parlament war parteipolitisch gespalten, da die Frage der Reduktion des $\mathrm{CO}_{2}$-Ausstoß bei Neuwagen auch eine Auseinandersetzung zwischen Industrie- und Umweltinteressen widerspiegelte. Die Grünen haben von Anfang bis Ende ausschließlich Umweltinteressen vertreten. Insbesondere für die deutschen Grünen war die deutsche Automobilindustrie der Hauptgegner. Aber auch die Sozialdemokraten haben den Umweltinteressen einen hohen Rang eingeräumt und sich für einen höheren Prozentsatz der schrittweisen Einbeziehung der Neuwagenflotte in die Gesetzgebung und höhere Strafzahlungen ausgesprochen. Auf der anderen Seite haben Liberale und Konservative in all diesen Punkten industrienahe Positionen bezogen. Diese ideologische Spaltung erklärt auch das Abstimmungsverhalten in den Ausschüssen.

Allerdings waren nationale Bruchlinien in gleicher Stärke vorhanden: Die nationale Automobilindustrie hat die jeweiligen Abgeordneten direkt oder über die nationalen Regierungen beeinflussen können. Die britischen Abgeordneten haben parteiübergreifend in einem gemeinsamen Änderungsantrag Ausnahmen für Nischenhersteller gefordert, die in erster Linie britischen Herstellern zugutekommen. Dies wurde auch von der britischen Regierung gefordert. Insbesondere liberale und konservative Abgeordnete aus Deutschland haben sich für die Anrechnung von Ökoinnovationen eingesetzt, ebenso wie die deutsche Regierung. Die Auseinandersetzung um die Lastenverteilung zeigte gleich doppelt die Wirksamkeit nationaler Interessen: Einerseits weil auch die Parlamentarier den deutsch-französisch/italienischen Gegensatz in der Lastenverteilung mit ihren Änderungsanträgen führten, andererseits aber, weil dieser Streit gerade durch die Einigung von Bundeskanzlerin Angela Merkel und Präsident Nicolas Sarkozy in den Hintergrund gerückt ist. Der deutsch-französische Kompromiss diente auch als Orientierungshilfe in punkto Strafzahlungen und Ökoinnovationen im Parlament.

Obwohl vor dem Hintergrund der Zerrissenheit des Parlaments in den Abstimmungen der Ausschüsse nicht von einem vereint auftretenden Parlament gesprochen werden kann, sollen zwei Elemente der Geschlossenheit hervorgehoben werden: Erstens hat das Parlament trotz Arbeitsüberlastung durch das Energie- und Klimapaket, des engen Zeitplans und den deutlichen Differenzen der Fraktionen eine Einigung mit dem Rat erreicht, was man als eine große Leistung bezeichnen kann. Zweitens hat das Parlament sich relativ früh auf das langfristige Ziel für 2020 geeinigt und dieses deutlich härter formuliert als in der deutsch-französischen Einigung vorgesehen. Es konnte sich mit dieser Forderung vereint gegen den Rat durchsetzen, wenngleich es dort ebenfalls Sympathien für ein fest fixiertes langfristiges Ziel von 95 $\mathrm{g} / \mathrm{km}$ gab. 
Das Resultat lautet, dass das Verhalten des Parlaments gleichermaßen national wie ideologisch motiviert war und ein geschlossenes Auftreten zur besseren Durchsetzung seiner Interessen gegenüber dem Rat erst nach langem Ringen zwischen den Fraktionen am Ende erfolgte. Der deutsch-französische Gipfel zwischen Präsident Nicolas Sarkozy und Bundeskanzlerin Angela Merkel erleichterte darüber hinaus die Einigung im Parlament indem er den Konflikt zwischen der deutschen und französischen Industrie im Hinblick auf die Lastenverteilung entschärfte und auch in den anderen Punkten Kompromisslinien darlegte.

Insgesamt zeigt der Entscheidungsprozess der $\mathrm{CO}_{2}$-Verordnung für Neuwagen trotz einiger Schwächen das Funktionieren des europäischen Entscheidungssystems auf, das auch unter massivem Zeitdruck tragfähige Lösungen hervorbringen kann. Die Akteure einigten sich schließlich auf einen Kompromiss, der den verschiedenen Interessen von Umwelt wie Industrie gerecht wurde, wenngleich das langfristige Ziel von 95 g im Jahr 2020 aus Industriesicht als sehr ehrgeizig bezeichnet werden muss.

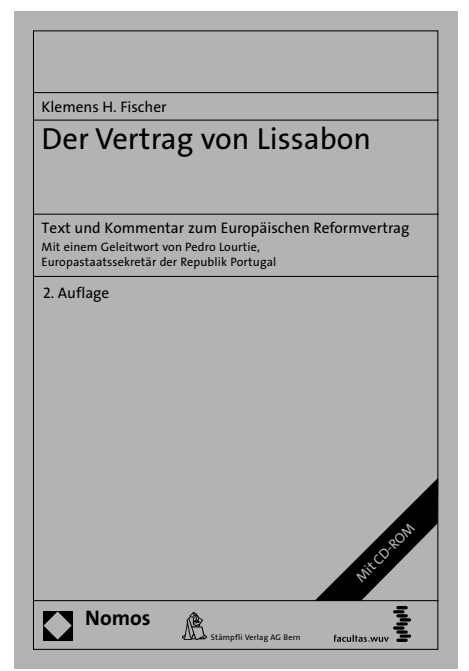

\section{Der Vertrag von Lissabon}

Text und Kommentar zum Europäischen Reformvertrag

Mit einem Geleitwort von Pedro Lourtie, Europastaatssekretär der Republik Portugal

Von PD Dr. Klemens H. Fischer

2. Auflage 2010, 623 S., brosch., mit CD-ROM, 79,-€, ISBN 978-3-8329-5284-6

Erscheint ca. Mai 2010

Am 1. Dezember 2009 ist der Vertrag von Lissabon endlich in Kraft getreten! Der vorliegende Kommentar, der in zweiter erweiterter Auflage erscheint, zeichnet nicht nur die Entstehungsgeschichte des Vertrags nach und erläutert die einzelnen Änderungen des EU-Primärrechts, sondern stellt in dieser zweiten Auflage auch den komplexen Ratifikationsprozess dar. Überdies wird erstmals für Wissenschaft und Praxis die konkrete Umsetzung des Reformvertrags detailliert vorgestellt, so beispielsweise die Neuerungen durch die Einführung eines Europäischen Auswärtigen Dienstes, die Einführung des ordentlichen Gesetzgebungsverfahrens, und die neuen Organe und Funktionsträger der Union.

Bitte bestellen Sie im Buchhandel oder versandkostenfrei unter $\bullet$ www.nomos-shop.de 